\title{
Proton Dominance of Sub-LET Threshold GCR SEE Rate
}

\author{
Rubén García Alía, Markus Brugger, Véronique Ferlet-Cavrois, Sytze Brandenburg, Jordan Calcutt, \\ Francesco Cerutti, Eamonn Daly, Alfredo Ferrari, Michele Muschitiello, Giovanni Santin, Slawosz Uznanski, \\ Marc-Jan van Goethem, Ali Zadeh
}

\begin{abstract}
-
We apply a Monte Carlo based integral rectangular parallelpiped (IRRP) approach to evaluate the impact of heavy ion reaction products on the Galactic Cosmic Ray (GCR) Single Event Effect (SEE) rate, concluding that owing to their similar high-energy $(>100 \mathrm{MeV} / \mathrm{n})$ SEE cross section and much larger abundance, protons are expected to be the dominating contributor. In addition, a broad set of components, ions and energies is used to explore the sub-LET threshold experimental region for standard ground-level heavy ion test energies, identifying an overall decreasing trend in the $10-80 \mathrm{MeV} / \mathrm{n}$ range due to the decreased contribution of complete and break-up fusion, and pointing out the limitations associated to the application of Monte Carlo SEE models in this energy interval.
\end{abstract}

\section{INTRODUCTION}

The importance of including nuclear interactions in Galactic Cosmic Ray (GCR) SEE rate calculations is treated in detail in [1] and references therein. Through Monte Carlo (MC) simulations and in-flight experimental data, the authors showed that, for a rad-hard component, not considering the indirect energy deposition events from nuclear interactions induced by ions with an insufficient Linear Energy Transfer (LET) to cause SEEs via direct ionization (hereafter referred to as subLET threshold ions) could lead to a two-order-of-magnitude underestimation of the in-flight SEE rate when compared to standard SEE prediction approaches excluding the contribution of heavy ion (HI) nuclear reactions. The studied component had a large LET threshold value and a significant proportion of high-Z material (namely tungsten) near its Sensitive Volume (SV).

In [2] however it is argued through a simple double-Weibull approach that the sub-LET threshold contribution to the GCR SEE rate for the case introduced above is actually limited to roughly $15 \%$ of the total value and that the disagreement between the standard approach and the in-flight data was in fact related to the use of a rectangular parallel-piped (RPP) approach instead of a more suitable intergral-RPP (IRPP) one.

In addition, in [3] it was shown that an IRPP-MC model that was highly successful in predicting the proton SEU cross

R. García Alía (ruben.garcia.alia@cern.ch), M. Brugger, F. Cerutti, A. Ferrari and S. Uznanski are with CERN, CH-1211, Genève, Switzerland.

V. Ferlet-Cavrois, J. Calcutt, E. Daly, A. Zadeh, M. Muschitiello and G. Santin are with the European Space Agency, ESTEC, 2200 AG Noordwijk, The Netherlands.

S. Brandenburg and M. J. van Goethem are with the University of Groningen, KVI-Center for Advance Radiation Technology (CART), Zernikelaan 25, 9747 AA Groningen, the Netherlands section based on the HI direct ionization response was however unable to reproduce the sub-LET threshold HI data with a similar level of accuracy. For some ions and energies the discrepancy was observed to be as large as two orders of magnitude, raising questions about the validity of applying MC calculations to estimate the impact of the sub-LET threshold region on the GCR SEE rate.

The work we present here first of all focuses on extending the IRPP-MC model benchmark with experimental data to a broader set of effects, components, ion species and energies. Despite the identified limitations, the model is considered suitable for its application to energies relevant to the GCR environments. Through an analysis of the respective energy deposition distributions and involved SEE cross sections, protons are found to be the dominating contributor to the subLET threshold region independently of the considered LET threshold. The implications of such observation in terms of SEE hardness assurance are discussed.

In addition, through a broad set of experimental data, we identify trends in the sub-LET threshold SEE cross section in the $10-80 \mathrm{MeV} / \mathrm{n}$ energy interval, comparing them with high-energy proton results and Monte Carlo nuclear code predictions. It was previously observed that the SEU cross section of a high-LET threshold SRAM increased significantly in the 10-40 MeV/n range [4] however the study is based on lighter ion data at lower energies, which can also contribute to the dependence attributed to an energy effect. In order to disentangle both effects, this work focuses on the energy dependence of the SEE cross section for a specific set of ions. In particular, ${ }^{12} \mathrm{C},{ }^{16} \mathrm{O}$ and ${ }^{20} \mathrm{Ne}$ are considered,

\section{HeAVy ION MEASUREMENTS}

\section{A. DUTs and test setups}

Six different components were tested in the scope of the sub-LET threshold study presented here. The test sample consists of 4 SRAMs (two for SEU and two for SEL) and two power MOSFETs tested for SEB, as detailed in Table I. All measurements reported in this work were performed at normal incidence.

The AT6 part was tested embedded in the ESA SEU Monitor, hosting 4 memory dies of the $0.25 \mu \mathrm{m}$ technology with a total size of $16 \mathrm{Mbit}$. The component has been exhaustively characterized in a broad range of facilities [5]-[8] and can therefore be used as a means of cross-calibrating the fluence and homogeneity thanks to the display of the physical location 
TABLE I

DEVICES STUDIED IN THIS WORK AND ASSOCIATED SEE TEST TYPE.

\begin{tabular}{c|c|c|c}
\hline Reference & Short Name & Type of Device & Type of Test \\
\hline \hline AT60142F & AT6 & SRAM & SEU \\
R1LV1616R & R1L & SRAM & SEU \\
BS62LV1600 & BS62 & SRAM & SEL \\
K6R4016VD1D & K6R & SRAM & SEL \\
SFR130 & SFR & Power MOSFET & SEB \\
STD10NF10 & STD & Power MOSFET & SEB \\
\hline
\end{tabular}

of the SEUs. For the results presented here, the "checkerboard" pattern was used, and the memory was only read at the end of each irradiation period.

The R1L component is a 16 Mbit SRAM built on a $0.15 \mu \mathrm{m}$ CMOS Thin-Film-Transistor (TFT) technology. Each bit cell uses two DRAM capacitors placed in the Back-End-Of-Line (BEOL) in order to decrease the memory's sensitivity to SEU [9]. As was shown in [10], its proton SEU cross section at roughly $200 \mathrm{MeV}$ is three orders of magnitude lower than the value for standard SRAMs of similar feature size. During the test, the memory was accessed through an FPGA in a so-called fill sequence consisting of checking for a given pattern (e.g. checker-board) and filling the inverse pattern every second. The address and read pattern of the words in error was logged and post-processed.

The SRAMs tested for SEL are built on a $0.18 \mu \mathrm{m}$ CMOS technology and were tested with a commercial de-latching system. A hold and cut time of $10 \mathrm{~ms}$ and $200 \mathrm{~ms}$ were set respectively, with a current alarm value of $10 \mathrm{~mA}$ for the BS62 (standby current consumption of $13 \mu \mathrm{A}$ ) and $20 \mathrm{~mA}$ for the K6R (standby current consumption of $1.7 \mathrm{~mA}$ ). The current consumption during the SEL events was limited to $100 \mathrm{~mA}$ in order to avoid potential destructive failures.

The power MOSFETs tested for SEB have a specified drainsource voltage $\left(\mathrm{V}_{\mathrm{DS}}\right)$ of $100 \mathrm{~V}$. Radiation tests were performed at the maximum specified $\mathrm{V}_{\mathrm{DS}}$ value and a gate-source voltage $\left(\mathrm{V}_{\mathrm{GS}}\right)$ of $0 \mathrm{~V}$. SEB test were performed in a non-destructive mode in order to collect a statistically meaningful number of events. An SEB event was identified by a sharp increase in the drain-source leakage current, $\left(\mathrm{I}_{\mathrm{DS}}\right)$. A value of $100 \mathrm{nA}$ was set as a detection threshold value, which compared to a background $\mathrm{I}_{\mathrm{DS}}$ value of $0.5-1 \mathrm{nA}$. As an output, the test program counted the number of SEBs and recorded the $V_{D S}$ profile of each event.

The de-lidding of the parts was performed through laser pre-decapsulation and chemical opening. The BEOL thickness and materials of the SRAMs were known either through information from the manufacturer or a Scanning Electron Microscopy (SEM) analysis and are reported in Table II along with other general information. As to what concerns the power MOSFETs, only the gate oxide, aluminum and polysilicon were present above the $12-15 \mu \mathrm{m}$ thick SV. Therefore, the degradation in energy and corresponding possible LET change before and in the SV for the experimental ions used in this work and introduced below is considered to be negligible.
TABLE II

SRAM CHARACTERISTICS INCLUDING BEOL THICKNESS AND MATERIALS. ALL SRAMS CELLS WERE OF THE 6T CMOS TYPE.

\begin{tabular}{c|c|c|c|c}
\hline SRAM & $\begin{array}{c}\text { Tech. node } \\
\left(\mu \mathrm{m}^{2}\right)\end{array}$ & $\begin{array}{c}\text { Cell Surface } \\
\left(\mu \mathrm{m}^{2}\right)\end{array}$ & $\begin{array}{c}\text { BEOL thick. } \\
(\mu \mathrm{m})\end{array}$ & Materials \\
\hline \hline AT6 & 0.25 & 10.0 & - & $3 \mathrm{Al}, 1 \mathrm{Cu}, 1$ Poly \\
BS62 & 0.18 & 5.40 & - & $2 \mathrm{Al}, 1$ Poly \\
K6R & 0.18 & 3.36 & 4.1 & $2 \mathrm{Al}, 1 \mathrm{~W}, 1$ Poly \\
R1L & 0.15 & 2.16 & 4.9 & $2 \mathrm{Al}, 1 \mathrm{~W}, 5$ Poly \\
\hline
\end{tabular}

TABLE III

PROPERTIES OF IONS USED AT UCL IN ORDER OF ASCENDING LET.

\begin{tabular}{c|c|c|c}
\hline Ion & $\begin{array}{c}\text { Energy } \\
(\mathrm{MeV} / \mathrm{n})\end{array}$ & $\begin{array}{c}\text { LET } \\
\left(\mathrm{MeVcm}^{2} / \mathrm{mg}\right)\end{array}$ & $\begin{array}{c}\text { Range(Si) } \\
(\mu \mathrm{m})\end{array}$ \\
\hline \hline${ }^{13} \mathrm{C}$ & 10.1 & 1.1 & 269 \\
${ }^{22} \mathrm{Ne}$ & 10.7 & 3.0 & 202 \\
${ }^{15} \mathrm{~N}$ & 4.0 & 3.3 & 60.4 \\
${ }^{20} \mathrm{Ne}$ & 3.9 & 6.4 & 46.3 \\
${ }^{40} \mathrm{Ar}$ & 9.5 & 10 & 121 \\
${ }^{40} \mathrm{Ar}$ & 3.8 & 15.9 & 41.1 \\
${ }^{58} \mathrm{Ni}$ & 10 & 20.4 & 101 \\
${ }^{83} \mathrm{Kr}$ & 9.4 & 32.3 & 94.2 \\
${ }^{84} \mathrm{Kr}$ & 3.6 & 40.4 & 41.1 \\
${ }^{124} \mathrm{Xe}$ & 3.4 & 67.7 & 38.5 \\
\hline
\end{tabular}

\section{B. Tests at UCL: focus on direct ionization cross section}

Heavy ion measurements were performed at the UCL facility [11] in March 2015 using both the high LET (E 4 MeV/n) and high penetration $(\mathrm{E} \sim 10 \mathrm{MeV} / \mathrm{n})$ cocktails. Measurements at UCL are performed in vacuum. The ions used for the tests reported in this paper are shown in Table III. Provided the large LET interval available as opposed to the relatively limited energy options, the focus of this test campaign was that of characterizing the direct ionization cross section (i.e. above the LET threshold). However, results considered below the LET threshold are treated together with the broader indirect ionization measurements collected at KVI-CART.

The SEE cross section results are shown in Figs. 1, 2 and 3 for the BS62, K6R and R1L parts respectively as a function of LET together with the corresponding Weibull fits for experimental data interpreted as being above threshold. We use a log-log scale in order to highlight the sub-LET threshold region.

When faced with a set of heavy ion results as a function of LET and in order to carry out a sub-LET threshold analysis as a function of ion species and energy, it is first of all necessary to differentiate the regions above (e.g. dominated by direct ionization) and below (e.g. dominated by nuclear reactions) the LET threshold. This can first of all be done through visual inspection by identifying the region in which points follow a Weibull-like trend and the interval below the sharp falloff in which a different, not necessarily regular behavior as a function of LET is observed (e.g. typically manifesting as an inflexion point towards the low end of the LET curve). However, this can be a complicated task, especially for data sets with few points involved. In addition, one cannot discard the possibility of having a response which is dominated by direct ionization overall but follows two different trends owing to e.g. a larger sensitivity over a smaller surface of the component. 
Alternatively, a more solid argument in order to determine the sub-LET threshold region is that of finding data points that, despite having similar LET values, show a very different cross section, as is the case for the K6R and R1L part (Figs. 2 and 3) at an LET of around $3 \mathrm{MeVcm}^{2} / \mathrm{mg}$. This is the case because, by definition and as long as the ionization track structure does not play an important role (as expected for the involved technologies), direct ionization effects are well described through the LET metric; whereas indirect ionization events are induced through nuclear reactions, whose probability and product characteristics will depend on the incident ion species and energy.

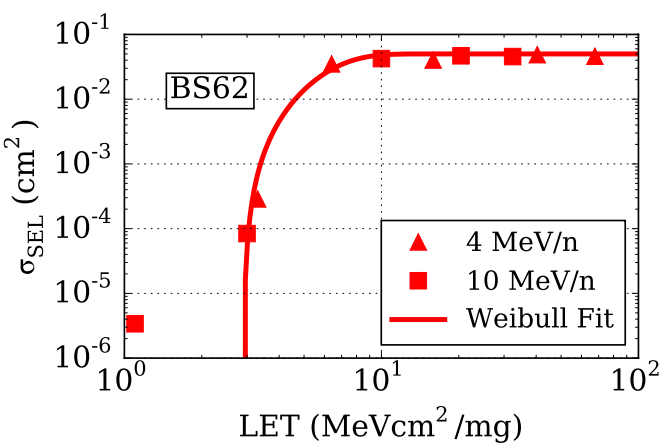

Fig. 1. Heavy ion SEL cross section for the BS62LV1600 memory at UCL.

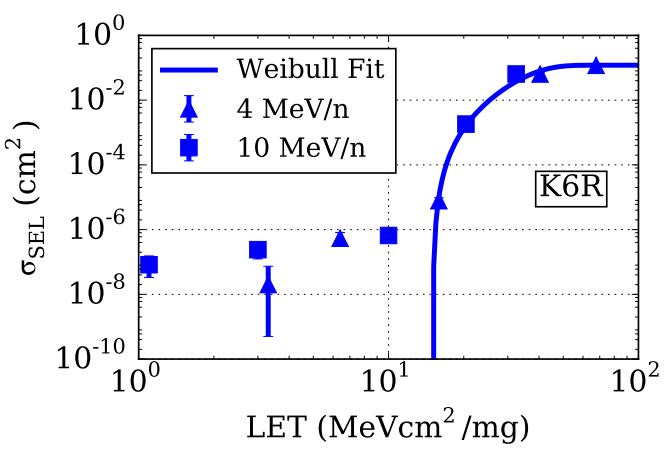

Fig. 2. Heavy ion SEL cross section for the K6R4016V1D memory at UCL.

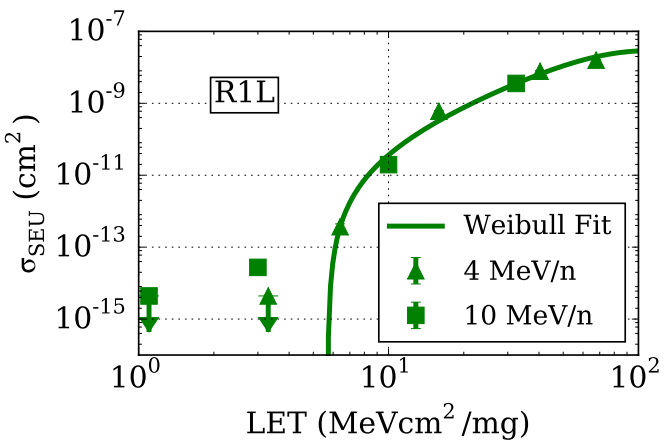

Fig. 3. Heavy ion SEU cross section for the R1LV1616R memory at UCL. Arrows pointing downwards correspond to $2 \sigma$ upper limits.
TABLE IV

WORD ERRORS FOR THE R1L TEST AT UCL DIVIDED BY THE MULTIPLICITY INTO SINGLE BIT UPSETS (SBU) AND MBUS OF DIFFERENT MULTIPLICITIES.

\begin{tabular}{c|c|c|c|c}
\hline $\begin{array}{c}\text { LET } \\
\left(\mathrm{MeVcm}^{2} / \mathrm{mg}\right)\end{array}$ & SBU & MBU2 & MBU3 & MBU4 \\
\hline \hline 67.7 & $1709(80.8 \%)$ & $406(19.1 \%)$ & 0 & 0 \\
40.4 & $1791(88.2 \%)$ & $239(11.8 \%)$ & 0 & 0 \\
32.2 & $826(96.5 \%)$ & $30(3.5 \%)$ & 0 & 0 \\
20.4 & $958(100 \%)$ & 0 & 0 & 0 \\
15.9 & $2049(99.9 \%)$ & $1(0.1 \%)$ & 0 & 0 \\
10.0 & $609(99.8 \%)$ & $1(0.2 \%)$ & 0 & 0 \\
6.4 & $103(97.2 \%)$ & $3(2.83 \%)$ & 0 & 0 \\
3 & $18(78.2 \%)$ & $3(13.0 \%)$ & $1(4.4 \%)$ & $1(4.4 \%)$ \\
\hline
\end{tabular}

In the case of SEU, the analysis of the Multiple Bit Upset (MBU) probability for the R1L component provides a complementary means of indirectly identifying the contribution of nuclear reactions as opposed to direct ionization. Though the logical-to-physical mapping of the memory was unknown, thus not allowing for a Multiple Cell Upset (MCU) analysis, we observed that at least a fraction of such events lead to MBUs, identified directly through the logical addressing of the memory. The words in error were categorized according to the number of SEUs in each word yielding the distribution shown in Table IV. As shown in [10], the percentage of MBUs (words with more than one bit in upset) and maximum multiplicity for a $480 \mathrm{MeV}$ proton beam were $16 \%$ and 4 respectively, therefore exhibiting a very similar trend to what is shown in Table IV for the LET value of $3 \mathrm{MeVcm}^{2} / \mathrm{mg}$ and thus clearly supporting the nuclear reaction hypothesis.

Though also tested at KVI-CART with the focus on the subLET threshold region, due to the observation of other effects such as stuck bits or an SEU cross section dependence with TID, the detailed analysis of the ion and energy dependence of the R1L component will be treated outside the scope of this paper.

As a summary of the direct ionization SEE cross sections measured at UCL, the parameters of the Weibull fits of the type shown in Eq. 1 can be found in Table V. For the AT6 component, a detailed description of the direct ionization HI response can be found in [3] where the LET threshold was determined to be $3.0 \mathrm{MeVcm}^{2} / \mathrm{mg}$. For the SFR power MOSFET, the HI data in the above-threshold region is too scarce to perform a fit to a Weibull function, however the experimental results point towards an LET threshold in the $5-8 \mathrm{MeVcm}^{2} / \mathrm{mg}$ interval. For the STD component, only lowLET measurements at KVI were performed and were assumed to be in the sub-LET threshold region.

$$
\sigma(\mathrm{L})=\sigma_{\text {sat }}\left[1-\exp \left(-\left(\frac{\mathrm{L}-\mathrm{L}_{\mathrm{o}}}{\mathrm{W}}\right)^{\mathrm{s}}\right)\right]
$$

\section{KVI-CART: focus on sub-LET threshold region}

KVI-CART [12] is a particularly suitable facility for subLET threshold studies owing to the large energies and fluxes obtainable when compared to other heavy ion facilities such as UCL or RADEF [13]. The maximum available energies (e.g. $80 \mathrm{MeV} / \mathrm{n}$ for carbon and oxygen and $30 \mathrm{MeV} / \mathrm{n}$ for neon) 
TABLE V

WEIBULL FIT PARAMETERS FOR THE DIRECT IONIZATION RESPONSE OF THE TWO MEMORIES. FOR THE R1L COMPONENT, THE $\sigma_{\text {sat }}$ VALUE IS IN UNITS OF $\mathrm{cm}^{2} /$ bit.

\begin{tabular}{c|c|c|c|c}
\hline SRAM & $\begin{array}{c}\sigma_{\text {sat }} \\
\left(\mathrm{cm}^{2}\right)\end{array}$ & $\begin{array}{c}\mathrm{L}_{\mathrm{o}} \\
\left(\mathrm{MeVcm}^{2} / \mathrm{mg}\right)\end{array}$ & $\begin{array}{c}\mathrm{W} \\
\left(\mathrm{MeVcm}^{2} / \mathrm{mg}\right)\end{array}$ & $\mathrm{s}$ \\
\hline \hline BS62 & $5.00 \times 10^{-2}$ & 2.9 & 3.79 & 1.90 \\
K6R & $1.20 \times 10^{-1}$ & 15 & 24.6 & 2.83 \\
R1L & $3.00 \times 10^{-8}$ & 5.7 & 60.4 & 2.54 \\
\hline
\end{tabular}

TABLE VI

DEGRADER CONFIGURATION AND ENERGY AND LET AT THE DUT LOCATION FOR HEAVY IONS USED AT KVI. THE DEGRADER MATERIAL WAS ALUMINUM EXCEPT FOR THE THICKNESS MARKED WITH THE STAR SUPERSCRIPT FOR WHICH POLYESTER WAS USED.

\begin{tabular}{c|c|c|c|c}
\hline Ion & $\begin{array}{c}\text { Degrader } \\
(\mathrm{mm})\end{array}$ & $\begin{array}{c}\text { Energy@DUT } \\
(\mathrm{MeV} / \mathrm{n})\end{array}$ & $\begin{array}{c}\text { LET@DUT } \\
\left(\mathrm{MeVcm}^{2} / \mathrm{mg}\right)\end{array}$ & $\begin{array}{c}\text { Range(Si)@DUT } \\
(\mathrm{mm})\end{array}$ \\
\hline \hline \multirow{3}{*}{${ }^{12} \mathrm{C}$} & 0.0 & 79.2 & 0.25 & 9.39 \\
& 4.5 & 51.3 & 0.34 & 4.33 \\
& 6.5 & 33.3 & 0.48 & 2.01 \\
& 7.7 & 17.4 & 0.81 & 0.637 \\
\hline \multirow{3}{*}{${ }^{16} \mathrm{O}$} & 0.0 & 72.4 & 0.47 & 3.55 \\
& 2.5 & 50.3 & 0.63 & 1.85 \\
& 4.0 & 32.1 & 0.92 & 0.842 \\
& 4.8 & 17.5 & 1.46 & 0.303 \\
\hline \multirow{2}{*}{$\mathrm{Ne} \mathrm{Ne}$} & 0.0 & 27.7 & 1.55 & 0.885 \\
& 0.5 & 15.2 & 2.50 & 0.317 \\
& 1.0 & 9.8 & 3.50 & 0.158 \\
\hline
\end{tabular}

enable the study of effects in an energy range significantly larger than that achieved up to $10 \mathrm{MeV} / \mathrm{n}$, as well as penetrations in the $\mathrm{mm}$ range. In addition, the relatively large fluxes (up to roughly $6 \times 10^{6} \mathrm{~cm}^{-2} \mathrm{~s}^{-1}$ for the considered run) allow for a statistically meaningful count collection even for cases with relatively low SEE cross sections (e.g. those typically associated to the sub-LET region).

Measurements at KVI were performed in two different test campaigns: the first in June 2015 and the second in March 2016. The beam intensity was monitored using a large ionization chamber calibrated against a $5 \mathrm{~mm}$ radius scintillator for each ion and energy.

Two different beam configurations were used, the first for a primary energy of $90 \mathrm{MeV} / \mathrm{n}$ for ${ }^{12} \mathrm{C}$ and ${ }^{16} \mathrm{O}$ ions and the second for ${ }^{20} \mathrm{Ne}$ with a primary energy of $30 \mathrm{MeV} / \mathrm{n}$. For the first, the field is produced using a $0.3 \mathrm{~mm}$ lead scatter foil $20 \mathrm{~cm}$ after the exit foil of the beam $(50 \mu \mathrm{m}$ Kevlar $\mathrm{C}_{14} \mathrm{H}_{10} \mathrm{~N}_{2} \mathrm{O}_{2}$ with a density of $1.44 \mathrm{~g} / \mathrm{cm}^{3}$ ), $3.2 \mathrm{~m}$ upstream from the Device Under Target (DUT). The energy of the ion on the DUT was calculated using the SRIM2013 simulation tool and considering the impact of the exit foil, air, scatter foil and (if present) degrader. The beam was measured to be homogeneous at a $90 \%$ level in a diameter of roughly $30 \mathrm{~mm}$.

The $30 \mathrm{MeV} / \mathrm{n}$ primary energy beam was dispersed using a $10 \mu \mathrm{m}$ thick gold foil $5 \mathrm{~m}$ from the DUT, which was placed $8 \mathrm{~cm}$ downstream the exit foil. The degraders were placed $20 \mathrm{~mm}$ upstream the DUTs. The beam was measured to be homogeneous at a $90 \%$ level in a diameter of roughly $20 \mathrm{~mm}$.

The resulting SEE cross sections as a function of energy are shown in Figs. 4, 5 and 6 for the different ions and DUTs considered, combining both UCL and KVI sub-LET threshold data. When concentrating on the ${ }^{12} \mathrm{C}$ and ${ }^{16} \mathrm{O}$ cross sections, a clear trend of decreasing cross section with energy is identified for all components, the latter being more pronounced in the case of the SEB cross section for the power MOSFETs (SFR and STD). Provided this observation holds for a broad range of effects and direct ionization responses, we interpret it as a result of a physical trend in the nuclear reactions involved as opposed to effect or technology related responses. In fact, as will be further developed in Section III with the aid of Monte Carlo calculations, this decrease is attributed to the decreasing fusion cross section and the increased fragmentation of the involved nuclei with increasing ion energy, compatible with what was shown in [14] both through measurements and simulations. It is also worth noting that, despite the fact that UCL tests are performed in vacuum and KVI experiments are in air and with the use of degraders, the transition of the SEE cross sections from $17 \mathrm{MeV} / \mathrm{n}$ (lowest KVI energy) down to $10 \mathrm{MeV} / \mathrm{n}$ (UCL) for the ${ }^{12} \mathrm{C}$ ion in Fig. 4 is very smooth. Therefore, the impact of the air and degraders on the measured SEE cross sections, though not considered explicitly in the simulations in Section III, can be indirectly interpreted as minor.

For the ${ }^{20} \mathrm{Ne}$ ion in Fig. 6 the situation is similar to that of the two lighter ions studied with one exception: whereas most components show a decrease in the SEE cross section between 10 and $28 \mathrm{MeV} / \mathrm{n}$, the AT6 device experiences an increase of a factor 5 between 15 ans $28 \mathrm{MeV} / \mathrm{n}$ which is not only in contrast with the rest of the DUTs but also with the ${ }^{12} \mathrm{C}$ and ${ }^{16} \mathrm{O}$ behavior for the same component. As shown in [3] a very similar value for the SEU cross section of the AT6 device was obtained independently for the same component at the TAMU facility using the $25 \mathrm{MeV} / \mathrm{n}$ ion cocktail, therefore it is unlikely that the result is due to an experimental artifact. In addition, one could argue that the LET of the $28 \mathrm{MeV} / \mathrm{n}^{20} \mathrm{Ne}$ ion $\left(1.6 \mathrm{MeVcm}^{2} / \mathrm{mg}\right)$ is close enough to the LET threshold (3.0 $\mathrm{MeVcm}^{2} / \mathrm{mg}$ ) to partially induce direct ionization SEUs, however in that case the point for the same ion at a lower energy $(15 \mathrm{MeV} / \mathrm{n})$ and larger LET $\left(2.5 \mathrm{MeVcm}^{2} / \mathrm{mg}\right)$ would show a larger SEU cross section, which is clearly not the case. Therefore, this anomalous behavior remains an open point subject to further analysis.

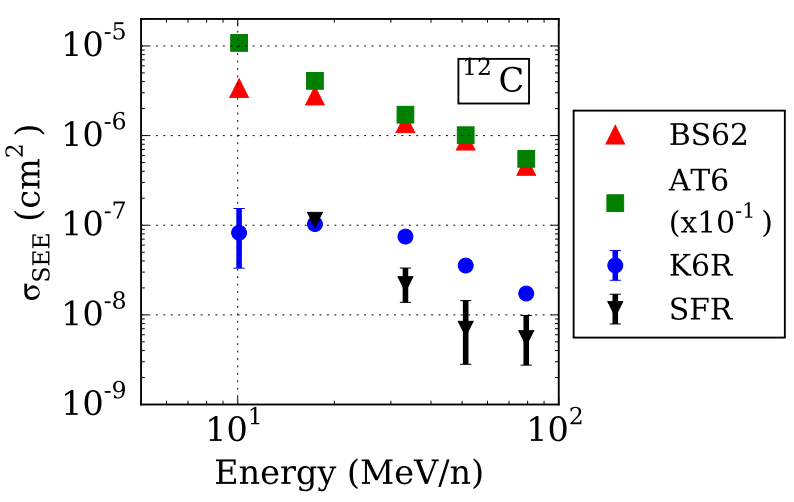

Fig. 4. Carbon sub-LET heavy ion cross section for the different components at UCL (10 MeV/n) and KVI-CART (rest of energies). Experiments at UCL were performed with ${ }^{13} \mathrm{C}$. 


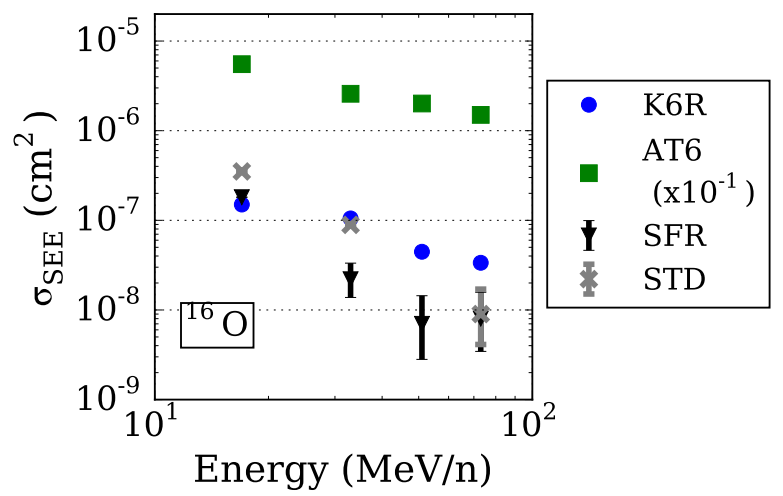

Fig. 5. ${ }^{16} \mathrm{O}$ sub-LET heavy ion cross section for the different components at KVI-CART.

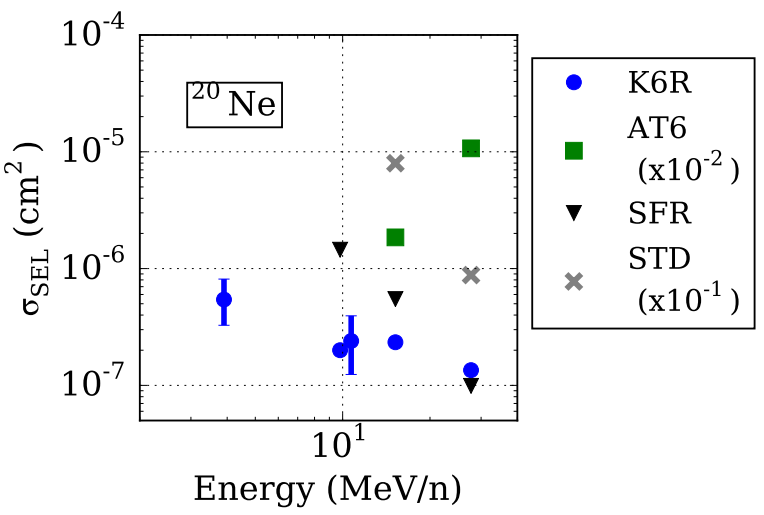

Fig. 6. ${ }^{20} \mathrm{Ne}$ sub-LET heavy ion cross section for the different components at KVI-CART. For the K6R component, results from UCL are also included and can be recognized by the relatively large error bars owing to the lower flux.

As to what regards the comparison between the sub-LET threshold heavy ion and the high-energy proton cross section data (the latter being an obvious example of nuclear reaction induced SEEs) Table VII shows the respective cross section values and ion-to-proton ratios. As can be observed, despite the hypothesis that both type of events are induced through indirect energy deposition, the heavy ion sub-LET threshold values are at least two orders of magnitude larger than highenergy proton ones.

TABLE VII

COMPARISON BETWEEN SUB-LET THRESHOLD HEAVY ION CROSS SECTION AND HIGH-ENERGY ( $200 \mathrm{MEV})$ PROTON CROSS SECTION. IN THE CASE OF THE BS62 COMPONENT, INSTEAD OF A 200 MEV PROTON BEAM, RESULTS FROM A 300 MEV QUASI-MONOENERGETIC BEAM AT RCNP ARE CONSIDERED. FOR THE SEU CROSS SECTION (AT6 AND R1L) CROSS SECTIONS ARE IN UNITS OF $\mathrm{cm}^{2} / \mathrm{bit}$.

\begin{tabular}{c|c|c|c|c}
\hline DUT & HI considered & $\begin{array}{c}\sigma_{\text {SEE }}^{\mathrm{HI}} \\
\left(\mathrm{cm}^{2}\right)\end{array}$ & $\begin{array}{c}\sigma_{\text {SEE }}^{\mathrm{P}} \\
\left(\mathrm{cm}^{2}\right)\end{array}$ & Ratio \\
\hline \hline AT6 & ${ }^{12} \mathrm{C} @ 10 \mathrm{MeV} / \mathrm{n}$ & $6.44 \times 10^{-12}$ & $2.60 \times 10^{-14}$ & $2.5 \times 10^{2}$ \\
R1L & ${ }^{20} \mathrm{Ne} @ 10 \mathrm{MeV} / \mathrm{n}$ & $2.74 \times 10^{-14}$ & $1.30 \times 10^{-17}$ & $2.1 \times 10^{3}$ \\
BS62 & ${ }^{12} \mathrm{C} @ 10 \mathrm{MeV} / \mathrm{n}$ & $3.39 \times 10^{-6}$ & $3.20 \times 10^{-8}$ & $1.1 \times 10^{2}$ \\
K6R & ${ }^{12} \mathrm{C} @ 10 \mathrm{MeV} / \mathrm{n}$ & $8.24 \times 10^{-8}$ & $4.40 \times 10^{-10}$ & $1.9 \times 10^{2}$ \\
STD & ${ }^{12} \mathrm{C} @ 17 \mathrm{MeV} / \mathrm{n}$ & $3.51 \times 10^{-7}$ & $1.50 \times 10^{-9}$ & $2.3 \times 10^{2}$ \\
\hline
\end{tabular}

\section{Simulations}

The simulation of the nuclear interaction in the sub-LET threshold region and subsequent energy deposition were calculated using the FLUKA Monte Carlo code [15]-[17]. FLUKA is a well benchmarked general purpose tool for the calculation of particle transport and interactions with matter, covering an extended range of applications such as electron and proton accelerator shielding, cosmic ray studies and medical physics.

In FLUKA, an adaptation of the DPMJET code [18] is used as nucleus-nucleus event generator for energies above $5 \mathrm{GeV} / \mathrm{n}$, with the evaporation stage of excited residual nuclei fully performed in FLUKA. For energies below $5 \mathrm{GeV} / \mathrm{n}$ and above $100 \mathrm{MeV} / \mathrm{n}$, FLUKA relies on a modified version of RQMD - 2.4 which is a Relativistic Quantum Molecular Dynamic code [19]. Respective results can be found in [20], [21]. At even lower energies $(<125 \mathrm{MeV} / \mathrm{n}$ in the developer version used in the scope of this work) a treatment based on the Boltzmann Master Equation (BME) has been implemented [22]. A benchmark of the associated ion-ion reaction cross sections in FLUKA and the properties of the fragments can be found in [23] in the context of carbon ion therapy. In addition, it is to be noted that elastic scattering for heavy ions is not implemented in FLUKA. Therefore, the potential impact of the associated recoil ions on the simulated SEE rate is not considered in this work.

In addition, the CRÈME MC online tool [24], [25] was used in an analogous way for comparison purpose. The online tool uses the Monte Carlo Radiative Energy Deposition (MRED) code [1] as a computational engine. MRED is based on $\mathrm{C}++$ code from Geant4 with additional Fotran components to simulate electron transport and nuclear reactions with high precision. The CEM03 and LAQGSM codes are used as nuclear physics codes for proton and heavy ion reactions respectively [26]-[28].

Simulations presented in the sections below were performed using the FLUKA (developer version 2015.1) and CRÈME MC (based on the mred-930 version) Monte Carlo tools.

\section{A. Calculations at a production level}

When analyzing the sub-LET threshold behavior for heavy ions, it is important to consider the analogy with the wellknown proton case. As in both scenarios SEEs are induced by a nuclear reaction between the projectile and a nucleus in the vicinity of the component's sensitive region, one of the first values to compare is the nuclear reaction cross section for protons and heavy ions at different energies.

The values were extracted using a set of tools in the FLUKA developer version and are shown in Table VIII. As can be seen, though HI reaction cross sections are indeed larger than proton ones, this is only by e.g. a factor $\sim 4$ when comparing ${ }^{12} \mathrm{C}$ at $10 \mathrm{MeV} / \mathrm{n}$ with protons at $100 \mathrm{MeV}$. Therefore, the SEE cross section ratios shown in Table VII cannot be justified simply by the enhanced probability of a heavy ion undergoing a nuclear interaction. In fact, this was the original argument provided in [29] against the interpretation of the low-LET heavy ion cross section plateau as being due to nuclear reactions. 
TABLE VIII

TOTAL NUCLEAR INELASTIC REACTION CROSS SECTION AS EXTRACTED FROM FLUKA FOR DIFFERENT IONS AND ENERGIES IN UNITS OF MB.

\begin{tabular}{c|c|c|c}
\hline Particle & $10 \mathrm{MeV} / \mathrm{n}$ & $100 \mathrm{MeV} / \mathrm{n}$ & $1 \mathrm{GeV} / \mathrm{n}$ \\
\hline \hline Proton & 885 & 459 & 456 \\
${ }^{12} \mathrm{C}$ & 1833 & 1318 & 1313 \\
${ }^{20} \mathrm{Ne}$ & 2103 & 1573 & 1568 \\
${ }^{56} \mathrm{Fe}$ & 2672 & 2350 & 2404 \\
\hline
\end{tabular}

In order to deepen the analysis, the characteristics of the nuclear reaction fragments need to be analyzed in addition to the probability of such a reaction occurring. We performed this study using FLUKA by scoring the Z, LET and energy distribution of the inelastic reaction products for different projectiles, targets and energies. The resulting Z-distributions for several selected cases are shown in Fig. 7. As can be seen, at large heavy ion energies (e.g. $200 \mathrm{MeV} / \mathrm{n}{ }^{12} \mathrm{C}$ ) the $\mathrm{Z}$-distribution of the fragments is very similar to that obtained with high-energy protons, with the exception of a significantly larger yield for projectile-like $\mathrm{Z}$ values in the case of the heavy ion. At lower heavy ion energies (e.g. $10 \mathrm{MeV} / \mathrm{n}$ ) there is a large probability that the projectile and target combine into a heavier nucleus through complete or break-up fusion. As is observed for ${ }^{12} \mathrm{C}$ on silicon and copper, the $\mathrm{Z}$ values of the fusion products largely depend on the projectile and target mass, and can reach values of the sum of their individual Z. Similar simulated results were shown in [30] for a ${ }^{124} \mathrm{Xe}$ $46 \mathrm{MeV} / \mathrm{n}$ beam on silicon.

Fig. 8 shows the nuclear product LET distribution for different carbon energies on silicon and compared to high energy protons. As can be seen, the behavior is similar to that observed for the $\mathrm{Z}$ distribution, with $200 \mathrm{MeV} / \mathrm{n}$ ions yielding a reaction product distribution similar to that of high-energy protons, and lower energy ions $(\sim 10 \mathrm{MeV} / \mathrm{n})$ generating higher LET values due to the larger mass of the fusion products. Therefore qualitatively speaking, one can expect the low-energy heavy ion sub-LET threshold SEE cross section to be significantly larger than that for protons, whereas as the heavy ion energy increases, the trend is towards a larger fragmentation, tending to a situation similar to that induced by protons.

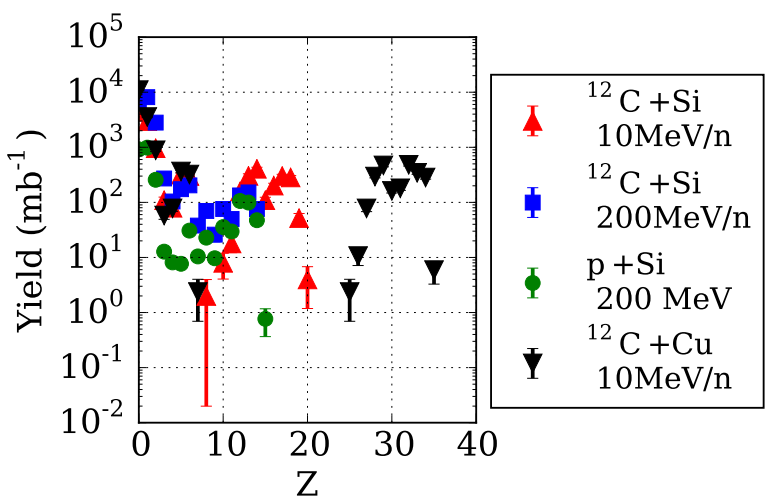

Fig. 7. Z-distribution of nuclear reaction products as calculated in FLUKA for different projectiles, targets and energies.

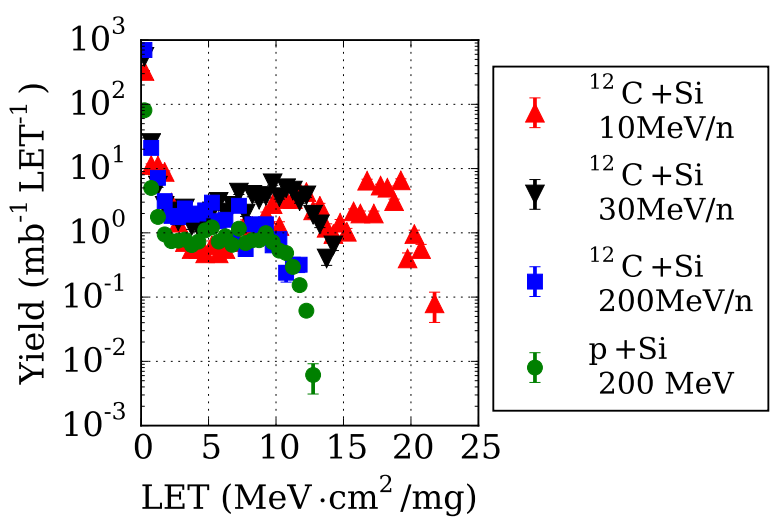

Fig. 8. LET-distribution of nuclear reaction products as calculated in FLUKA for different projectiles and energies.

\section{B. SEL model and comparison with experimental data}

Once the nuclear reaction product properties have been studied, the next step in the analysis is that of performing a full transport simulation where the energy deposition is scored in the relevant SV. In order to do so we use an IRPP-MC model to extract the expected sub-LET threshold SEL cross section for the experimental results presented in Section II. As explained in further detail in [3], [31] the approach is based on the (i) the definition of an RPP SV and its surroundings (ii) the MC simulation of the energy deposition distribution in the SV for the ion species and energies of interest and (iii) the convolution of the obtained distributions with the experimental HI direct ionization response function (thus the name IRPP, in analogy with the analytic approach that convolves the LET spectrum in space with the experimental heavy ion cross section [32]).

The relevant model input values and their respective justification are the following:

- SEL SV dimensions of $20 \times 4 \times 2 \mu \mathrm{m}^{3}$ as typically considered through laser studies of similar components ( [33] and references therein)

- A BEOL thickness of $5 \mu \mathrm{m}$ composed of $\mathrm{SiO}_{2}$

- A tungsten layer of $122 \mathrm{~nm}$ placed $200 \mathrm{~nm}$ above the SVs. Considering an SRAM cell size of $2 \times 2 \mu \mathrm{m}^{2}$ for the technologies studied in this paper, this corresponds to a volume of roughly $0.5 \mu \mathrm{m}^{3}$ per cell, as determined in a construction analysis of SRAM K6R [34]

This IRPP-MC model was successfully used to the extract the proton SEL cross section from the HI response for a broad number of SRAM memories in [35], where the K6R and BS62 devices treated here corresponds to SRAM D and SRAM F respectively.

The model introduced above was used to calculate the energy deposition distributions for the ion and energies tested at UCL and KVI-CART and the response functions defined in Eq. 1 and Table V were applied for both SRAMs considered. The resulting calculated cross section values are compared with the experimental data for carbon in the $10-80 \mathrm{MeV} / \mathrm{n}$ range in Fig. 9. As can be seen, whereas the model very successfully reproduces the energy dependence for the BS62 memory, it fails in doing so for the K6R part. In particular at 
$10 \mathrm{MeV} / \mathrm{n}$ the experimental results is over a factor 10 larger than the calculated value.

We (partially) attribute the mismatch between the simulated and experimental data at $10 \mathrm{MeV} / \mathrm{n}$ to the combined impact of (i) the strong dependence of the $\mathrm{Z}$ and LET fusion product distributions on the projectile and target mass (see Fig. 7) and (ii) the fact that the LET threshold for the K6R component is large $\left(15 \mathrm{MeVcm}^{2} / \mathrm{mg}\right)$ and corresponds to the maximum LET value of silicon in silicon, therefore the impact of heavier products is expected to be dominant. As the BEOL of the component is only represented in a very approximative way, the exact composition and location of the metalization, insulation and interconnect layers is expected to play a crucial role in the simulated low-energy heavy ion cross sections for ions whose fusion mass with silicon yields products only slightly above the LET threshold. This interpretation is supported by Fig. 10 which shows the simulated and measured cross section values for the $\mathrm{K} 6 \mathrm{E}$ as a function of the ion atomic number $(\mathrm{Z})$ for an energy of $10 \mathrm{MeV} / \mathrm{n}$. As can be seen, the agreement between experimental value and calculation for the neon $(Z=10)$ and $\operatorname{argon}(Z=20)$ cases is within a factor 3 and therefore significantly more satisfactory than the carbon $(Z=6)$ case at this energy. We attribute this to their larger masses which yield an energy deposition distribution less dependent on the surrounding materials and LET threshold value.

In addition, it is worth noting that differences between the two Monte Carlo codes considered were significantly more pronounced in the $10-80 \mathrm{MeV} / \mathrm{n}$ interval than at larger energies for cases dominated by high- $Z$ fragments, as we will later show. Likewise, in FLUKA an abrupt SEE cross section change was observed at $125 \mathrm{MeV} / \mathrm{n}$ for the high-LET threshold case (K6R) which we interpret as related to the decrease in the energy transferred to the heavy fragments in the transition from the energy range treated through the Boltzmann Master Equation (BME) [22] to that in which an RQMD (Relativistic Quantum Molecular Dynamic code) is used [20], [21].

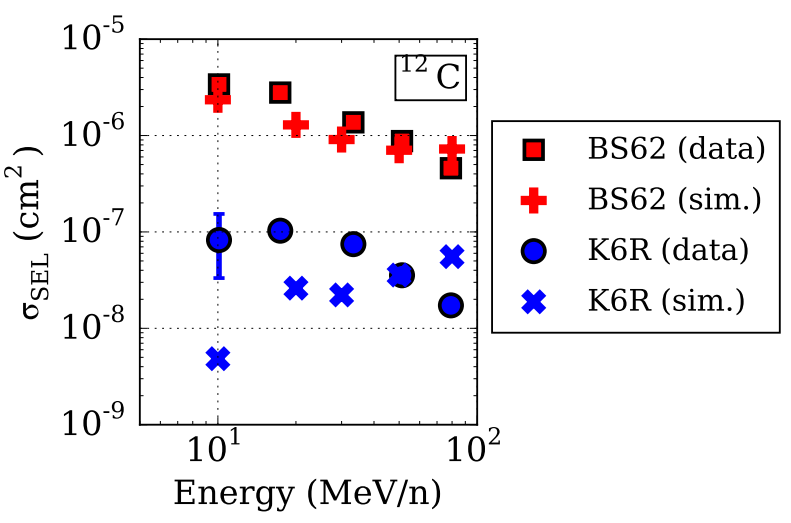

Fig. 9. Simulated carbon sub-LET heavy ion SEL cross section for both components in the $10-80 \mathrm{MeV} / \mathrm{n}$ range compared to experimental data.

\section{Effect of high-Z materials at energies above $100 \mathrm{MeV} / \mathrm{n}$}

Despite the fact that the introduced model fails to reproduce the experimental data for carbon at $10 \mathrm{MeV} / \mathrm{n}$, we consider it applicable to larger energies because (i) it does successfully

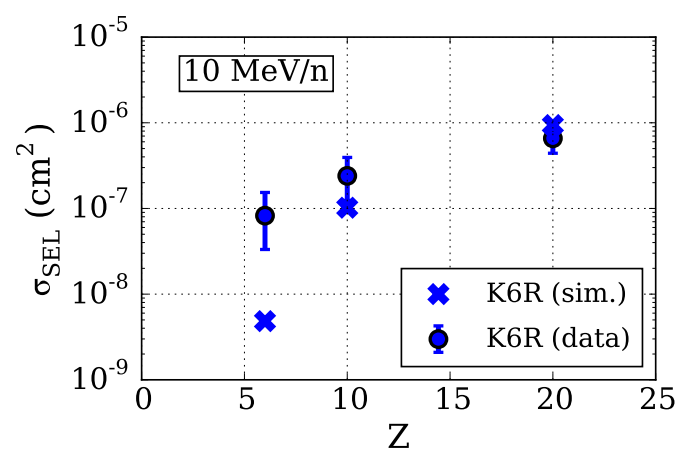

Fig. 10. Simulated $10 \mathrm{MeV} / \mathrm{n}$ sub-LET heavy ion cross section for the K6R part as a function of atomic number compared to experimental data.

reproduce the cross section at $10 \mathrm{MeV} / \mathrm{n}$ for heavier ions (ii) it is successful in reproducing the carbon results in the $30-80 \mathrm{MeV} / \mathrm{n}$ within a factor 3 and (iii) a similar SEU model was capable of reproducing the iron sub-LET threshold cross section in the 200-1500 MeV/n again with a factor 3 [3], thus providing confidence in terms of applying it to larger energies than those tested for experimentally in this work, but still highly relevant in the GCR environment. In addition and as discussed in Section III-A, the fragment properties of high-energy $(>100 \mathrm{MeV} / \mathrm{n})$ heavy ions is similar to the welldescribed proton case.

The application of the model to larger energies is performed using the K6R model, both with and without the W-layer described above. The resulting calculated cross section values are shown in Fig. 11 for the ${ }^{12} \mathrm{C}$ and protons with the $\mathrm{W}$ layer in the $100 \mathrm{MeV} / \mathrm{n}-100 \mathrm{GeV} / \mathrm{n}$ range. In this interval, the respective ${ }^{12} \mathrm{C}$ cross section without the $\mathrm{W}$-layer is roughly two orders of magnitude smaller that near $10 \mathrm{MeV} / \mathrm{n}$, similarly to what was observed experimentally for the silicon-dominated SEU case in [3] for the ${ }^{56} \mathrm{Fe}$ in the $200 \mathrm{MeV} / \mathrm{n}-1.5 \mathrm{GeV} / \mathrm{n}$ interval. As can be seen, though in the several tens of $\mathrm{MeV} / \mathrm{n}$ range the cross section behavior is very different between protons and heavy ions, the value in the GCR-dominant energy interval is similar.

In addition, results using the CRÈME MC tool are also presented, showing an agreement with FLUKA within a factor two, significantly better than in the several tens of $\mathrm{MeV} / \mathrm{n}$ range in which both codes differ significantly.

In order to have a closer look at the effect of the considered high-Z material near the SV in our model, we plot the reverse integral of the energy deposition distribution as a function of the volume-equivalent LET threshold. The volume-equivalent LET is defined as the deposited energy divided by the SV thickness and material density. As can be seen in Fig. 12, for LET threshold values above roughly $10 \mathrm{MeVcm}^{2} / \mathrm{mg}$ the presence of tungsten clearly results in a larger calculated cross section. In the same plot we have included the curve corresponding to $1 \mathrm{GeV}$ protons for the case with tungsten. It is worth noting that despite the very different projectile masses, the maximum energy deposited by the fragments is the same for protons and heavy ions (in this case, $Z=6$ ), therefore suggesting that the properties of the involved fission fragments 


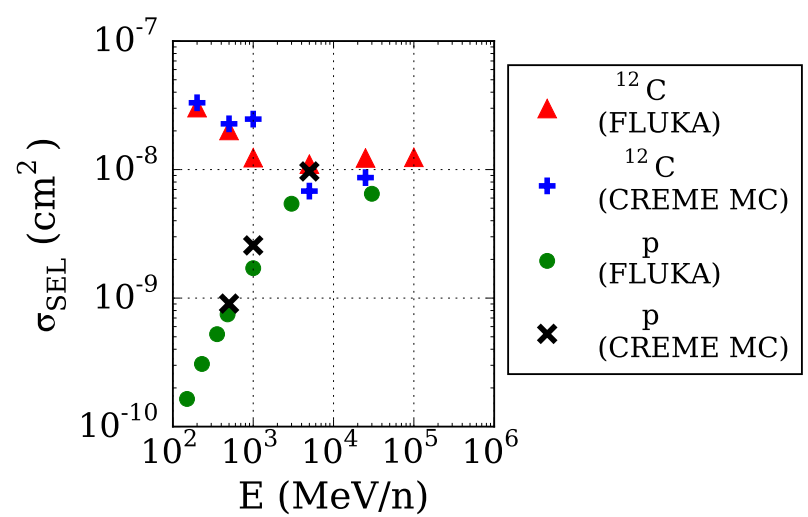

Fig. 11. Simulated sub-LET heavy ion cross section for the ${ }^{12} \mathrm{C}$ ion and protons using the K6R model.

very weakly depend on the nature of the original particle but are rather intrinsic to the involved fissile material.

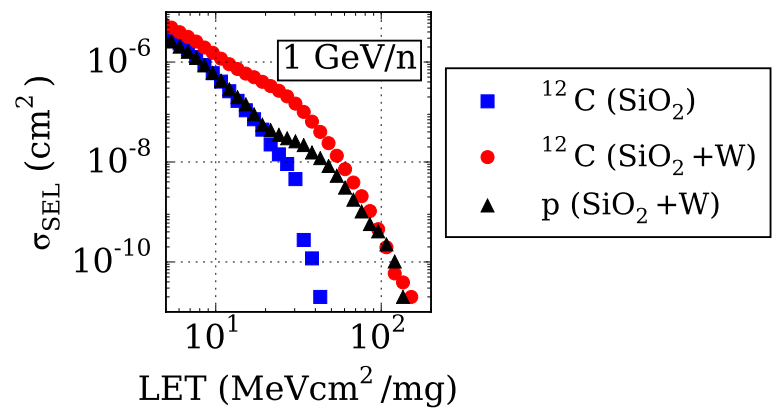

Fig. 12. Reverse integral of the energy deposition distribution for the RPP geometry introduced model at $1 \mathrm{GeV} / \mathrm{n}$ for protons (including the $\mathrm{W}$ layer) and carbon (both with and without the W layer)

Despite the very similar maximum energy deposited at $1 \mathrm{GeV} / \mathrm{n}$ by both protons and carbon ions, Fig. 12 also shows that the associated cross section for components with large LET threshold values (roughly above $10 \mathrm{MeVcm}^{2} / \mathrm{mg}$ ) will result in a larger cross section in the case of carbon than for protons. In order to quantify this, the simulated $1 \mathrm{GeV} / \mathrm{n}$ SEL cross section for the K6R response function is shown in Fig. 13 for different $Z$ values using both FLUKA and CRÈME MC. As can be seen, the $1 \mathrm{GeV} / \mathrm{n}$ cross section (which in the case of heavy ions and in analogy to what is shown in Fig. 11 for carbon is also expected to be the saturation value) has a very weak dependency on the ion atomic number above $\mathrm{Z}=6$. We will exploit this result in the following subsection in order to estimate and compare the contribution of each particle (protons, helium and heavy ions) to the expected sub-LET SEL rate in a GCR environment.

\section{Impact on sub-LET threshold GCR SEE rate}

In order to evaluate the relative sub-LET threshold impact of the different heavy ions for the K6R calculation, we consider

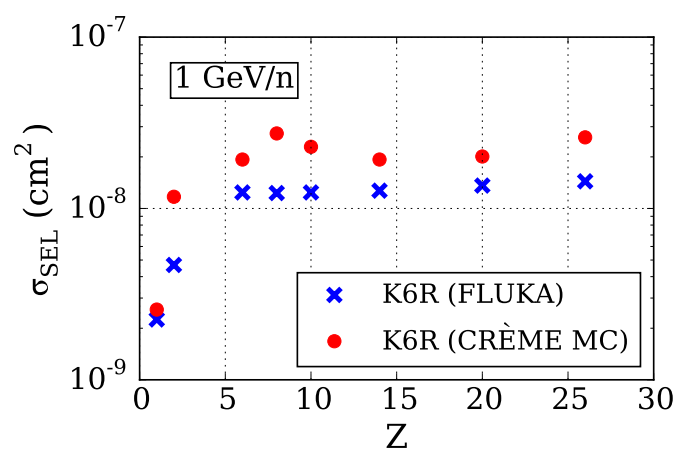

Fig. 13. Calculated $1 \mathrm{GeV} / \mathrm{n}$ SEL cross section for the K6R model and different ions using FLUKA and CRÈME MC.

a constant SEL cross section above $100 \mathrm{MeV} / \mathrm{n}$ and obtain the associated sub-LET threshold SEL rate by multiplying the integral flux above this energy times the $1 \mathrm{GeV} / \mathrm{n}$ cross section values shown in Fig. 13 for FLUKA. As an example, 95\% of the carbon GCR flux is above this energy, thus justifying the fact of neglecting the sub-LET threshold contribution from lower energies, despite the potentially larger SEE cross sections due to fusion products.

In the following, we make the distinction between protons, helium and heavy ions $(2<\mathrm{Z}<29)$. As protons have a strong dependence with energy up to roughly $3 \mathrm{GeV} / \mathrm{n}$, we use the Weibull fit to the simulated data and fold it with the respective flux instead of assuming a constant cross section. It is possible that heavy ions lighter than carbon (such as helium) also exhibit a significant energy dependence below $1 \mathrm{GeV} / \mathrm{n}$, however assuming a constant cross section value at this energy is considered as a valid approximation.

The resulting fluxes (extracted using the CREME96 model for solar minimum and 100 mils of aluminum), simulated 1 $\mathrm{GeV} / \mathrm{n}$ SEL cross sections and calculated sub-LET threshold SEL rates for the K6R model are shown in Table IX. As can be seen, protons are expected to clearly dominate the subLET threshold rate. This is due to the fact that despite their associated $1 \mathrm{GeV} / \mathrm{n}$ cross section being a factor $\sim 5$ smaller than for heavy ions, their much larger flux (roughly $90 \%$ of the total) largely compensates for it. In addition it is to be noted that using the same RPP dimensions and HI response function, the expected direct ionization SEL rate for the K6R component was calculated to be $1.18 \times 10^{-2}$ events/day using CREME96. Therefore, according to our calculations the sub-LET threshold contribution to the GCR SEL rate would only represent roughly $10 \%$ of the total value and would therefore not justify the need of including it in the SEE rate prediction tools. However, this value could significantly change for different SV dimensions, HI response and high-Z material content.

Nevertheless, the conclusion that in relative terms protons will dominate the sub-LET threshold SEE rate is independent of the specific component, as it is a result of the larger proton flux and similar fission cross section and properties of the associated high-LET products. 
TABLE IX

INTEGRATE FLUX ABOVE $100 \mathrm{MEV/N}$, CALCULATED 1 GEV/N CROSS SECTION AND ESTIMATED SUB-LET THRESHOLD SEL RATE FOR THE DIFFERENT PARTICLE TYPES.

\begin{tabular}{c|c|c|c}
\hline Particle & $\begin{array}{c}\phi_{>100 \mathrm{MeV} / \mathrm{n}} \\
\left(\mathrm{cm}^{-2} \mathrm{day}^{-1}\right)\end{array}$ & $\begin{array}{c}\sigma_{\mathrm{SEL}}^{\text {sat. }} \\
\left(\mathrm{cm}^{2}\right)\end{array}$ & $\begin{array}{c}\text { SEL Rate } \\
\left(\mathrm{day}^{-1}\right)\end{array}$ \\
\hline \hline Proton & $3.75 \times 10^{5}$ & $2.25 \times 10^{-9}$ & $1.11 \times 10^{-3}(85 \%)$ \\
Helium & $3.36 \times 10^{4}$ & $4.68 \times 10^{-9}$ & $1.57 \times 10^{-4}(12 \%)$ \\
HI & $3.22 \times 10^{3}$ & $1.24 \times 10^{-8}$ & $3.99 \times 10^{-5}(3 \%)$ \\
\hline
\end{tabular}

\section{SUMmARY AND CONCLUSIONS}

The extensive experimental analysis in this work shows that, for a broad set of components, effects and ions, the SEE cross section in the several tens of $\mathrm{MeV}$ range tends to decrease with energy. Through Monte Carlo simulation, this is attributed to the combined effect of a decrease in the fusion probability and an increase in the fragmentation of the nuclei, both resulting in lower LET products and energy deposition events. In addition, we also implement and benchmark a Monte Carlo approach to be used to extrapolate the results to $\mathrm{GeV} / \mathrm{n}$ energies, hardly accessible experimentally but dominating the GCR environment.

The main conclusion of the work we present here is that protons are expected to dominate the sub-LET threshold SEE rate for a GCR environment. The calculation that $1 \mathrm{GeV} / \mathrm{n}$ protons and heavy ions interacting with high-Z materials yield similar energy deposition distributions allows for the generalization of this observation to a broader set of components than those specifically considered here. It is important to note however that despite the expected proton sub-LET dominance, SEE tests with energies corresponding to peak GCR fluxes remain of interest for various reasons, including for example potential effects related to the ionization track structures [36], [37], or enabling access to SVs in complex components (e.g. flip-chips) and systems for which standard heavy ion energies $(\sim 10 \mathrm{MeV} / \mathrm{n})$ are not penetrating enough [38].

In addition, we showed that for the K6R component - for which tungsten proved to play a predominant role in the proton SEL cross section - it is expected that the overall sub-LET contribution to the total GCR SEL rate is below 10\%. However, this could vary for devices with a larger LET threshold (e.g. above the iron knee at $\sim 30 \mathrm{MeVcm}^{2} / \mathrm{mg}$ ) or a larger proportion of high-Z materials near the SV (e.g. using gold packages [39]) for which high-Z fragments with larger LET values could potentially dominate the total SEE rate. Likewise, newer technologies or different SV dimensions could also result in a stronger impact of the sub-LET threshold region on the overall SEE rate. In any case, the conclusion that the sub-LET threshold rate is expected to be dominated by protons relaxes the Radiation Hardness Assurance (RHA) constrains, as access to high-energy heavy ion beams is typically costly and restricted.

Finally, the comparison between experimental data and the IRPP-MC model output for the low energy sub-LET threshold region shows that in general and especially for certain cases (e.g. ${ }^{12} \mathrm{C}$ ion for the high-LET threshold K6R model at $10 \mathrm{MeV} / \mathrm{n}$ ) the agreement is significantly less satisfactory than in the case of protons. We consider that, despite they are not expected to have a dominating impact on the total GCR SEE rate, a deeper understanding of the HI sub-LET threshold behavior at low energies is relevant in order to improve the interpretation of ground HI testing. For instance, interpreting sub-LET threshold data as a result of direct ionization could lead to significantly underestimating the LET threshold and thus overestimating the in-flight SEE rate.

\section{REFERENCES}

[1] R. A. Reed, R. A. Weller, M. H. Mendenhall, D. M. Fleetwood, K. M. Warren, B. D. Sierawski, M. P. King, R. D. Schrimpf, and E. C. Auden, "Physical processes and applications of the Monte Carlo radiative energy deposition (MRED) code," IEEE Trans. Nucl. Sci., vol. 62, no. 4, pp. 1441-1461, Aug 2015.

[2] E. Petersen, Single event effects in aerospace. John Wiley \& Sons, 2011, ch. 10 .

[3] R. G. Alia, C. Bahamonde, S. Brandenburg, M. Brugger, E. Daly, V. Ferlet-Cavrois, R. Gaillard, S. Hoeffgen, A. Menicucci, S. Metzger, A. Zadeh, M. Muschitiello, E. Noordeh, and G. Santin, "Sub-LET threshold SEE cross section dependency with ion energy," IEEE Trans. Nucl. Sci., vol. 62, no. 6, pp. 2797-2806, Dec 2015.

[4] P. E. Dodd, J. R. Schwank, M. R. Shaneyfelt, J. A. Felix, P. Paillet, V. Ferlet-Cavrois, J. Baggio, R. A. Reed, K. M. Warren, R. A. Weller, R. D. Schrimpf, G. L. Hash, S. M. Dalton, K. Hirose, and H. Saito, "Impact of heavy ion energy and nuclear interactions on single-event upset and latchup in integrated circuits," IEEE Trans. Nucl. Sci., vol. 54, no. 6, pp. 2303-2311, Dec. 2007.

[5] R. Harboe-Sorensen, F.-X. Guerre, and A. Roseng, "Design, testing and calibration of a Reference SEU Monitor system," in Radiation and Its Effects on Components and Systems, 2005. RADECS 2005. 8th European Conference on, Sept. 2005, pp. B3-1 -B3-7.

[6] R. Harboe-Sorensen, C. Poivey, F.-X. Guerre, A. Roseng, F. Lochon, G. Berger, W. Hajdas, A. Virtanen, H. Kettunen, and S. Duzellier, "From the reference SEU monitor to the technology demonstration module onboard Proba-II," IEEE Trans. Nucl. Sci., vol. 55, no. 6, pp. $3082-3087$, Dec. 2008.

[7] R. Harboe-Sorensen, C. Poivey, N. Fleurinck, K. Puimege, A. Zadeh, F.-X. Guerre, F. Lochon, M. Kaddour, L. Li, D. Walter, A. Keating, A. Jaksic, and M. Poizat, "The technology demonstration module on-board Proba-II," IEEE Trans. Nucl. Sci., vol. 58, no. 3, pp. $1001-1007$, June 2011.

[8] R. Harboe-Sorensen, C. Poivey, A. Zadeh, A. Keating, N. Fleurinck, K Puimege, F.-X. Guerre, F. Lochon, M. Kaddour, L. Li, and D. Walter, "PROBA-II technology demonstration module in-flight data analysis," IEEE Trans. Nucl. Sci., vol. 59, no. 4, pp. 1086-1091, Aug 2012.

[9] Y. Kihara, Y. Nakashima, T. Izutsu, M. Nakamoto, Y. Konishi, and T. Yoshihara, "A 16M SRAM with improved characteristics using DRAM technology," in Asian Solid-State Circuits Conference, 2005, Nov 2005, pp. 17-20.

[10] S. Uznanski, R. G. Alia, E. Blackmore, M. Brugger, R. Gaillard, J. Mekki, B. Todd, M. Trinczek, and A. Villanueva, "The effect of proton energy on SEU cross section of a 16 Mbit TFT PMOS SRAM with DRAM capacitors," IEEE Trans. Nucl. Sci., vol. 61, no. 6, pp. 3074 3079, Dec 2014.

[11] "Centre du Recherches du Cyclotron, Université Catholique de Louvainla-neuve, Belgium.” [Online]. Available: http://www.cyc.ucl.ac.be/

[12] "KVI - Center for Advanced Radiation Technology." [Online]. Available: http://www.rug.nl/kvi-cart

[13] A. Virtanen, R. Harboe-Sorensen, A. Javanainen, H. Kettunen, H. Koivisto, and I. Riihimaki, "Upgrades for the radef facility," in Radiation Effects Data Workshop, 2007 IEEE, vol. 0, July 2007, pp. 38-41.

[14] N. A. Dodds, R. A. Reed, M. H. Mendenhall, R. A. Weller, M. A. Clemens, P. E. Dodd, M. R. Shaneyfelt, G. Vizkelethy, J. R. Schwank, V. Ferlet-Cavrois, J. H. Adams, R. D. Schrimpf, and M. P. King, "Charge generation by secondary particles from nuclear reactions in BEOL materials," IEEE Trans. Nucl. Sci., vol. 56, no. 6, pp. 3172-3179, Dec 2009.

[15] A. Ferrari, P. R. Sala, A. Fasso, and J. Ranft, "FLUKA, a multiparticle transport code," CERN-2005-10-2005, INFN/TC-05/11, SLAC$R-773,2005$.

[16] T. Böhlen, F. Cerutti, M. Chin, A. Fassò, A. Ferrari, P. Ortega, A. Mairani, P. Sala, G. Smirnov, and V. Vlachoudis, "The FLUKA code: developments and challenges for high energy and medical applications," Nuclear Data Sheets, vol. 120, pp. 211-214, 2014. 
[17] "Overview of the FLUKA code," Annals of Nuclear Energy, vol. 82, pp. $10-18,2015$.

[18] S. Roesler, R. Engel, and J. Ranft, "The Monte Carlo event generator DPMJET-III," in Advanced Monte Carlo for Radiation Physics, Particle Transport Simulation and Applications. Springer, 2001, pp. 1033-1038.

[19] H. Sorge, H. Stöcker, and W. Greiner, "Poincaré invariant hamiltonian dynamics: modelling multi-hadronic interactions in a phase space approach," Annals of Physics, vol. 192, no. 2, pp. 266-306, 1989.

[20] V. Andersen, F. Ballarini, G. Battistoni, M. Campanella, M. Carboni, F. Cerutti, A. Empl, A. Fasso, A. Ferrari, E. Gadioli et al., "The FLUKA code for space applications: recent developments," Advances in Space Research, vol. 34, no. 6, pp. 1302-1310, 2004.

[21] H. Aiginger, V. Andersen, F. Ballarini, G. Battistoni, M. Campanella, M. Carboni, F. Cerutti, A. Empl, W. Enghardt, A. Fassò et al., "The FLUKA code: New developments and application to $1 \mathrm{GeV} / \mathrm{n}$ iron beams," Advances in Space Research, vol. 35, no. 2, pp. 214-222, 2005.

[22] F. Cerutti, G. Battistoni, G. Capezzali, P. Colleoni, A. Ferrari, E. Gadioli, A. Mairani, and A. Pepe, "Low energy nucleus-nucleus reactions: the BME approach and its interface with FLUKA," in Proceedings of the 11th International Conference on Nuclear Reaction Mechanisms, Varenna (Italy), 2006.

[23] T. T. Böhlen, F. Cerutti, M. Dosanjh, A. Ferrari, I. Gudowska, A. Mairani, and J. M. Quesada, "Benchmarking nuclear models of FLUKA and GEANT4 for carbon ion therapy," Physics in Medicine and Biology, vol. 55, no. 19, p. 5833, 2010. [Online]. Available: http://stacks.iop.org/0031-9155/55/i=19/a=014

[24] R. A. Weller, M. H. Mendenhall, R. A. Reed, R. D. Schrimpf, K. M. Warren, B. D. Sierawski, and L. W. Massengill, "Monte Carlo simulation of single event effects," IEEE Trans. Nucl. Sci., vol. 57, no. 4, pp. 1726$1746,2010$.

[25] M. H. Mendenhall and R. A. Weller, "A probability-conserving cross-section biasing mechanism for variance reduction in Monte Carlo particle transport calculations," Nuclear Instruments and Methods in Physics Research Section A: Accelerators, Spectrometers, Detectors and Associated Equipment, vol. 667, pp. 38 - 43, 2012. [Online]. Available: http://www.sciencedirect.com/science/article/pii/S0168900211021541

[26] S. Mashnik, A. Sierk, K. Gudima, and M. Baznat, "CEM03 and LAQGSM03 - new modeling tools for nuclear applications," in Journal of Physics: Conference Series, vol. 41, no. 1. IOP Publishing, 2006, p. 340 .

[27] S. Mashnik, K. Gudima, N. Mokhov, and R. Prael, "LAQGSM03. 03 upgrade and its validation," arXiv preprint arXiv:0709.1736, 2007.

[28] S. Mashnik, K. Gudima, R. Prael, A. Sierk, M. Baznat, and N. Mokhov, "CEM03. 03 and LAQGSM03. 03 event generators for the MCNP6, MCNPX, and MARS15 transport codes," arXiv preprint arXiv:0805.0751, 2008.

[29] R. Ecoffet, S. Duzellier, D. Falguere, L. Guibert, and C. Inguimbert, "Low LET cross-section measurements using high energy carbon beam [DRAMs/SRAMs]," IEEE Trans. Nucl. Sci., vol. 44, no. 6, pp. 22302236, Dec 1997.

[30] V. Ferlet-Cavrois, J. R. Schwank, S. Liu, M. Muschitiello, T. Beutier, A. Javanainen, A. Hedlund, C. Poivey, A. Mohammadzadeh, R. HarboeSorensen, G. Santin, B. Nickson, A. Menicucci, C. Binois, D. Peyre, S. K. Hoeffgen, S. Metzger, D. Schardt, H. Kettunen, A. Virtanen, G. Berger, B. Piquet, J.-C. Foy, M. Zafrani, P. Truscott, M. Poizat, and F. Bezerra, "Influence of beam conditions and energy for SEE testing," IEEE Trans. Nucl. Sci., vol. 59, no. 4, pp. 1149-1160, Aug 2012.

[31] R. G. Alia, M. Brugger, S. Danzeca, V. Ferlet-Cavrois, C. Poivey, K. Roed, F. Saigne, G. Spiezia, S. Uznanski, and F. Saigne, "Energy dependence of tungsten-dominated SEL cross sections," IEEE Trans. Nucl. Sci., vol. 61, no. 5, pp. 2718-2726, Oct 2014.

[32] J. C. Pickel, "Single-event effects rate prediction," IEEE Trans. Nucl. Sci., vol. 43, no. 2, 1996.

[33] Y.-T. Yu, J.-W. Han, G.-Q. Feng, M.-H. Cai, and R. Chen, "Correction of single event latchup rate prediction using pulsed laser mapping test," IEEE Trans. Nucl. Sci., vol. 62, no. 2, pp. 565-570, April 2015.

[34] R. Alia, M. Brugger, S. Danzeca, V. Ferlet-Cavrois, C. Frost, R. Gaillard, J. Mekki, F. Saigne, A. Thornton, S. Uznanski, and F. Wrobel, "SEL hardness assurance in a mixed radiation field," IEEE Trans. Nucl. Sci., vol. 62, no. 6, pp. 2555-2562, Dec 2015.

[35] R. G. Alia, E. W. Blackmore, M. Brugger, S. Danzeca, V. FerletCavrois, R. Gaillard, J. Mekki, C. Poivey, K. Roed, F. Saigne, G. Spiezia, M. Trinczek, S. Uznanski, and F. Wrobel, "SEL cross section energy dependence impact on the high energy accelerator failure rate," IEEE Trans. Nucl. Sci., vol. 61, no. 6, pp. 2936-2944, Dec 2014.

[36] M. Bagatin, S. Gerardin, A. Paccagnella, and V. Ferlet-Cavrois, "Single and multiple cell upsets in 25-nm NAND flash memories," IEEE Trans. Nucl. Sci., vol. 60, no. 4, pp. 2675-2681, Aug 2013.

[37] G. Hubert, P. L. Cavoli, C. Federico, L. Artola, and J. Busto, "Effect of the radial ionization profile of proton on seu sensitivity of nanoscale srams," IEEE Trans. Nucl. Sci., vol. 62, no. 6, pp. 2837-2845, Dec 2015.

[38] H. Quinn, "Challenges in testing complex systems," IEEE Trans. Nucl. Sci., vol. 61, no. 2, pp. 766-786, April 2014.

[39] T. L. Turflinger, D. A. Clymer, L. W. Mason, S. Stone, J. S. George, M. Savage, R. Koga, E. Beach, and K. Huntington, "RHA implications of proton on gold-plated package structures in SEE evaluations," IEEE Trans. Nucl. Sci., vol. 62, no. 6, pp. 2468-2475, Dec 2015. 